\title{
Discussing the Efficacy and Safety of Covid-19 Vaccine Available in India-
}

\section{A Mini Review}

\section{Yogendra Shrestha, ${ }^{*}$ Rajesh Venkataraman, ${ }^{2}$ Jeet Bahadur Moktan, ${ }^{1}$ Renukaradhya Chitti, ${ }^{1}$ Shiv Kumar Yadav. $^{1}$}

1. Research Scholar, Department of Pharmacy Practice, Sri Adichunchanagiri College of Pharmacy, Adichunchanagiri University, BG Nagara-571448, India.

2. Head, Department of Pharmacy Practice, Sri Adichunchanagiri College of Pharmacy, Adichunchanagiri University, BG Nagara-571448, India.

Corresponding Author: Dr.Yogendra Shrestha

Email ID: dryogendrastha@gmail.com

\begin{abstract}
:
Background: Many variants detected after Wuhan-Hu-1 reference which were able to develop the resistance against the neutralizing antibodies induced by vaccine and may cause false negative results in diagnostic test. Novel variant B1.617 was detected in India and the Covid-19 cases hiked to its maximum; forcing the government towards approving a new vaccine for restricted use in emergency situation to cover a maximum population.
\end{abstract}

Aims: This review looks at the efficacy, safety, and economical aspects of vaccines that have been authorized in India.

Materials and methods: Wide-ranging assessment and analysis of accessible resources on online database.

Results: The rAd26-s \& rAd5-s demonstrate high efficacy as well as safety, followed by BBV152 and AZD1222. Various combinations of the vaccines with different platforms or vectors may induce wide range of immunity than a specific one. As per economical aspect, AZD1222 is more economical than the other two currently approved in India.

Conclusion: There is a lack of clear end point to measure efficacy of the vaccine so the epidemiological studies with huge number of populations is required which may predict the perfect endpoint for efficacy measurement. Until then, inoculation with locally accessible 
vaccines and self-awareness about disease transmission prevention are the main options for reducing fatalities, protecting the health-care system, and eventually disease control.

Key Words: Covid-19, Immunization, Vaccine Efficacy, Vaccine Safety.

\section{Highlight}

- The goal of vaccination is to reduce deaths, protect the health care system and finally disease control.

- All the vaccine made for immunization were targeting Wuhan-Hu-1 reference virus, after that lots many variants were detected which developed resistance against vaccines available for immunization.

- It is required to carry out the epidemiological studies to establish a clear endpoint for vaccine efficacy measurement.

- Wearing mask, maintaining distance, regular hand washing and avoiding crowed is still the best option to prevent the SARS-CoV-2 infection even after vaccination.

\section{Introduction:}

Novel Severe Acute Respiratory Syndrome Coronavirus 2 (SARS-CoV-2), which is responsible for Coronavirus disease (Covid-19) has spread to 220 countries and has been found to be challenging. ${ }^{1}$ As of $10^{\text {th }}$ November 2021, globally there were 250.90 million laboratory-confirmed cases and more than 5.065 million fatalities. In India there were 34.388 million laboratory-confirmed cases and more than 4.16 lakhs fatalities. ${ }^{2}$

After SARS-CoV-2 was initially infected through the nose, the virus quickly spread to the lungs, using Angiotensin-converting enzyme (ACE) 2 as binding receptor to invade the epithelial cell.$^{3,4,5}$ If the immune system fails to fight SARS-CoV-2 at this stage, the virus moves down to the lungs, where it can develop to fatal levels. ${ }^{6}$ The ACE- 2 receptor can be found on the surface of a variety of pulmonary and extrapulmonary cell types, including cardiac, renal, intestinal, and pancreatic cells, endothelial cells ${ }^{7}$ which may be the reason for severe condition in comorbid patients.

The reproduction number $\left(\mathrm{R}_{0}\right)$ rate of the virus is very high: 2.24-3.58; can survive on different surfaces for up to 72 hours or more. ${ }^{8}$ So far, SARS-CoV-2 has acquired new mutations at the rate of $\sim 2$ changes per month. ${ }^{9}$ At present, compared with the Wuhan-Hu-1 reference, each new sample has about ten mutation sites on average. The variant B.1.1.7, 
which was identified for the first time in the UK, may trigger false negative results in diagnostic tests which target the S gene. ${ }^{10}$ Recently new variant is detected in India, denoted B1.617, thought to be more contagious. ${ }^{11}$ The mutation is caused by random replication errors or due to the host's defense mechanism (called RNA editing). ${ }^{9}$ Which make virus more harmful to the host, rapid spread and complicated to find a targeted medicine or vaccine.

As of 22 April 2021, 91 and 184 vaccines are in clinical and preclinical development, respectively. ${ }^{12}$ The target of vaccine research is to obtain direct evidence of vaccine effectiveness against SARS-CoV-2 infection in humans. Candidate vaccines against SARSCoV-2 may fight infection, disease, or spread, and vaccines that capable to reduce any of these components may help disease control. ${ }^{13}$ The US Food and Drug Administration (FDA) has approved the distribution and use of three vaccines in the US: BNT162b2 vaccine by Pfizer-BioNTech on December 11, 2020, mRNA-1273 vaccine by Moderna on December 18, 2020, and ad26.cov2.s (Janssen Covid Vaccine) by Johnson \& Johnson on February 27, 2021, Unfortunately, the Janssen Covid vaccine was halted on April 23, 2021, after six people who were inoculated with it developed a rare and serious form of blood clot. ${ }^{14}$ The Drug Controller General of India has approved three vaccines for restricted use in emergency situation in the India: the ChAdOx1 nCoV-19 (AZD1222@) vaccine (AZD1222) by Oxford-AstraZeneca, BBV152 vaccine (BBV152®) by Bharat Biotech on $3^{\text {rd }}$ January 2021 and Gam-COVID-Vac RAd26-s \& rAd5-s® by Gamaleya Research Institute of Epidemiology and Microbiology 15,16

In this review, we will look at the efficacy, safety, and cost of the Covid-19 vaccines that have been approved by the DCGI in India for limited use in emergency situations.

\section{Methodology:}

Various databases (ScienceDirect, PubMed/Medline, Scopus, Google Scholar, Nature, and Cochrane Database of Systematic Reviews) were searched until April 2021. The search strategy employed were "rAd26 and rAd5 and SARS-Cov-2 or Covid-19 or nCoV", “AZD1222 or ChAdOx1 nCoV-19 and SARS-Cov-2 or nCoV or Covid-19”, "BBV152 and SARS-Cov-2 or Covid-19 or nCoV", "BNT162b2 mRNA and SARS-Cov-2 or Covid-19 or nCoV", "mRNA-1273 and SARS-Cov-2 or Covid-19 or nCoV", "SARS-Cov-2 variants", "Covid Vaccines and ADR". 


\section{Platforms of Covid-19 Vaccine:}

Covid-19 vaccines are developed from various sources. Virus vaccines, nucleic acid vaccines, viral vector vaccines, and protein-based vaccines are among the most important. ${ }^{17,18,19}$ The virus in a viral vaccine should be entirely inactivated and related to the risk of disease transmitted by the vaccine. DNA and RNA fragments stored in plasmids are used in nucleic acid vaccines. When viral proteins are taken up by host cells, they can be generated. ${ }^{17,20}$ Viral vector-based vaccines use viral vectors to introduce genetic sequences encoding antigens into host cells. However, the genetic material encoding the antigen may be lost during the manufacturing process, which may cause the vaccine to fail. The virus protein subunits are isolated and assembled into virus-like particles (VLP) using recombinant methods, which can imitate the structure of wild viruses and elicit a strong immune response, but are not contagious due to a lack of genetic material. ${ }^{17,18,19}$

\section{Effectiveness of vaccine:}

Vaccine trials should prove useful vaccine efficacy in preventing serious diseases, and protection time should be evaluated. The efficacy of vaccine can be evaluated against "infection, severity, and/or shedding/ transmission" keeping as an end point, and those who can able to demonstrate $\sim 50 \%$ point estimate can be acceptable as a Covid-19 vaccine (according to WHO). ${ }^{21}$ It appears to be nonspecific, reflecting the complexity of evaluating the clinical efficacy of candidate vaccine. ${ }^{22}$ As per the FDA, laboratory verified SARS-CoV2 infection is a good primary endpoint for vaccine efficacy trials, with a $50 \%$ chance of success in a placebo-controlled trial. ${ }^{23}$ Recently identified variants are showing false negative results in diagnostic test. ${ }^{10}$ Also, it may not be an appropriate end point to estimate vaccine efficacy.

Appropriate endpoints may only be predicted in epidemiological studies conducted after the vaccine has been widely deployed. Until we can use the vaccine's ability to prevent infection, disease, hospital admission, intensive care unit admission, death and transmission as the end points for efficacy measurement. ${ }^{22}$

\section{Vaccines authorized in India:}


Globally, many vaccines are available some of them are AZD1222® (Oxford-AstraZeneca), mRNA-1273 (Moderna), Janssen (Johnson \& Johnson), AZD1222 (Pfizer BioNTech), RAd26-s \& rAd5-s V® (Gamaleya), BBV152® (Bharat Biotech), CoronaVac (Sinovac), NVX-Cov2373 (Novavax), BBIBP-CorV (Sinopharm) etc. In India, DCGI has approved three vaccines for restricted use in emergency situation in the India: the AZD1222@, BBV152®, and RAd26-s \& rAd5-sV®.$^{15,16}$ As on $25^{\text {th }}$ April, around 140.91 million vaccines have been inoculated ${ }^{24}$ which is around $10 \%$ of total population.

\section{AZD1222®:}

The ChAdOx1 nCoV-19 vaccine (AZD1222) is made up of the replication-deficient simian (Chimpanzee) adenovirus vector ChAdOx1, which carries the full-length structural surface glycoprotein of SARS-CoV-2 along with a tissue plasminogen activator leader sequence. ChAdOx1 nCoV-19 expression optimizes the coding sequences for the codon of the spike protein. Two doses are required to administer at a dose of $0.5 \mathrm{ml}$, contain $3.5-6.5 \times 10^{10}$ viral particles as a single intramuscular injection (IM) into the deltoid up to 12 weeks apart (target 4 weeks) which induced maximum humoral and cellular immune responses against SARS$\mathrm{CoV}-2{ }^{25,26,27}$ It can be kept in the refrigerator at temperatures ranging from $+2^{\circ} \mathrm{C}$ to $+8^{\circ} \mathrm{C}$. When opened, multi-portion vials should be used as soon as practically possible and within 6 hours if held between $2{ }^{\circ} \mathrm{C}$ to $25^{\circ} \mathrm{C}$. ${ }^{28}$ It has shown the general efficacy of $70.42 \%$ in primary analysis population (Licensing regimen + Exploratory analysis) in the trials carried out in the UK and Brazil. ${ }^{26,28}$ Same vaccine shows only $22 \%$ efficacy according to preliminary South African data. It might be the reason of new variant 501Y.V2 (B.1.351), which is resistant to both natural and vaccine-induced immunity. ${ }^{29}$ Due to the South Africa variant (B.1.351), Shabir AM et al. found just 10.4 percent vaccine effectiveness and vaccine did not provide defense against mild-moderate Covid-19. However, the vaccine's effectiveness against the UK strain (B.1.1.7) and Indian strain (B.1.617) of SARS-CoV-2 is comparable to that of other lineages. ${ }^{30,31}$ So it may not be effective in new variants. It is available at rate Rs. 400/(5.36\$) for state government and Rs. 600/- (8.04\$) for private hospital per dose. ${ }^{32}$ Injection site tenderness (>60\%); injection site discomfort, headache, exhaustion (>50\%); myalgia, malaise (>40\%); pyrexia, chills (>30\%); and arthralgia, nausea (>20\%) were the most commonly recorded unfavorable reactions. The majority of adverse reactions were mild to moderate in intensity and apparently went away after a few days. By day 7 the incidence of subjects with at least one local or systemic reaction was $4 \%$ and $13 \%$, respectively. As compared to the first dose, the second dose's adverse reactions were milder and were 
recorded less frequently. ${ }^{25,27,28}$ In the case report reported by Marie S et al., anti-PF4 antibodies were observed 6 to 24 days after receiving the first dose of AZD1222, unrelated to the use of heparin therapy, in a case report of 23 mostly young, usually healthy patients who presented with atypical thrombosis, mainly involving cerebral veins, and thrombocytopenia.. ${ }^{33}$ With the exception of one patient who presented with fatal intracranial haemorrhage, Andreas G et al. confirmed that 11 original patients starting 5 to 16 days after vaccination presented with one or more thrombotic events; nine had cerebral venous thrombosis, three had splanchnic-vein thrombosis, three had pulmonary embolism, and four had other thromboses; six of these patients died. ${ }^{34}$ Chatterjee S et al. has reported a case of myocardial infraction post vaccination after 2 days. ${ }^{35}$ National Adverse event following immunization (AEFI) has documented which include myocardial infraction, cardiac death, trigger pro-thrombotic state, cardiovascular event, hypertensive emergency and anaphylaxis as adverse events in 11 patients and among them 10 had lost their lives. ${ }^{36}$ Although a causal link has yet to be established, viral vector, a vaccine additive, or a flaw in the manufacturing process may all play a role. ${ }^{33,37}$

\section{BBV152@:}

The virus strain (NIV-2020-770) with the Asp614Gly mutation, which was isolated and sequenced from a Covid-19 patient, was used to produce BBV152. It is an entire virion $\beta$ propiolactone-inactivated SARS-CoV-2 vaccine with a toll-like receptor (TLR) 7/8 agonist molecule absorbed to alum (Algel-IMDG). To induce full cell mediate response, it injects intramuscularly in to deltoid muscle at a volume of $0.5 \mathrm{ml} /$ dose in two-dose regimen 28 days apart. It can be stored in the refrigerator at temperatures ranging from $+2^{\circ} \mathrm{C}$ to $+8^{\circ} \mathrm{C}$, which is ideal for immunization cold chains. A Phase 3 clinical trial with 25,800 participants is ongoing, with interim analysis results indicating vaccine efficacy of 81 percent. ${ }^{38,39,40}$ Sapkal GN et al. study shown that this vaccine is effective against B1.1.7 and B.1.617 variants. ${ }^{31,41}$ It is available at the rate of Rs. 600/- (8.04\$) for state government and Rs. 1200/- (16.08\$) for private hospital. ${ }^{42}$ Pain and swelling at the injection site were listed as local adverse effects, while fever, weakness or malaise, myalgia, body aches, headache, nausea or vomiting, anorexia, chills, generalized rash, and diarrhea were listed as systemic adverse events. ${ }^{38,39,40}$ AEFI had document 2 adverse event associated with vaccination which include sweeting, dizziness, anxiety, cold extremities, hypotension and anaphylaxis. ${ }^{36}$ Detail phase 3 clinical trial report will be revealed imminently, which will give more data with respect to the immunization efficacy and undesirable impact of it. 


\section{RAd26-s \& rAd5-s®:}

Recombinant adenovirus type $26(\mathrm{rAd} 26)$ and recombinant adenovirus type 5 (rAd5), both of which bear the gene for SARS-CoV-2 full-length glycoprotein S, are included in the vaccine (rAd26-s and rAd5-s). ${ }^{43,44}$ For both recombinant adenoviruses, a complete dose of the vaccine contain $10^{10}$ or $10^{11}$ viral particles ${ }^{45}$ and given intramuscularly one followed by another with 21 days apart. The antigen transmitted by adenoviral vectors is known to induce both cellular and humoral immunity after a single immunization, making it useful as an emergency pandemic prevention method. A long-lasting immune response can be achieved by combining two immunizations. It is available in two formulation, frozen and lyophilized dry powder vaccine. For injection per dose, frozen vaccine inoculated at a volume of $0.5 \mathrm{~mL}$ and lyophilized dry powder vaccine must be reconstituted in $1 \mathrm{~mL}$ of sterile water. Frozen vaccine required $-18^{\circ} \mathrm{C}$ and lyophilized dry powder vaccine can storage at $+2{ }^{\circ} \mathrm{C}$ to $+8^{\circ} \mathrm{C}$. It has shown $91.6 \%$ vaccine efficacy. ${ }^{43,44,45}$ It is equally effective in case of B1.1.7 and B.1.617 variant and in case of B1.351 variant it has shown only minimal efficacy but better than other available vaccines. ${ }^{46,47,48,49}$ It is expected to be accessible in India by end of May 2021 at the rate less than $10 \$$ per dose. ${ }^{50,51}$ Immunization with this vaccine is linked to mild adverse events such as discomfort at the injection site (58\%), hyperthermia $(50 \%)$, headache $(42 \%)$, asthenia (28\%), muscle and joint pain (28\%). There were no severe adverse events identified. $^{43,44}$

\section{Discussion:}

Based on aspects discussed throughout this manuscript; there is no clear end point to measure efficacy of the vaccines, different studies use various endpoints such as laboratory confirmed SARS-CoV-2 infection, severity, and/or shedding/ transmission. ${ }^{21,23}$ The epidemiological studies with huge number of populations may predict the perfect endpoint for efficacy measurement. As per the available data (Table no. 1), the efficacy of RAd26-s \& rAd5-s® is comparatively high i.e., 91.6\% followed by BBV152® with $80 \%$ and AZD1222® $70.42 \%$ efficacy after successful inoculation of 2 doses as per respective protocol, all of these vaccines had shown their effectiveness to avert UK variant. But in case of South African variant, RAd26-s \& rAd5-s® has shown minimal efficacy, AZD1222® is less effective and the data for BBV152® is scarcely available. This efficacy against SARS-CoV-2 variants was 
discovered in laboratory studies using serum from infected patients with a small sample size, necessitating further tests with a larger number of human participants to determine the exact efficacy against new variants.

National AEFI has detected till date, 13 serious adverse events among which, 11 were from AZD1222® and 2 from BBV152® inoculation, out of 13- 10 lost their lives following vaccination with AZD1222® and 3 people recovered after hospitalization (1 of AZD1222® and 2 of BBV152®). ${ }^{35}$ AZD1222® vaccinated subjects have shown pain in the injection site, fatigue, headache, myalgia, pyrexia, chills, arthralgia as a mild and myocardial infraction, cerebra venous thrombosis, thrombocytopenia as a severe adverse event. ${ }^{25,27,28,36}$ Similarly, BBV152® vaccinated subjects have shown pain in injection site, fatigue, headache, myalgia, pyrexia, chills, arthralgia, cold extremities as mild and hypotension, anaphylaxis as a severe adverse event. ${ }^{36,38,39,40}$ Still RAd26-s \& rAd5-s ${ }^{\circledR}$ is not available in India, data shared from clinical trial and other data from different countries has shown no serious adverse events, only mild adverse events were injection site pain, headache, myalgia, asthenia, pyrexia and arthralgia. $^{43,44}$

Storage and transportation of vaccines has been a quite challenging task, the storage units and Temperature monitoring device (TMD) requires regular monitoring to ensure proper vaccine stability and function, maintaining the temperature at $+2{ }^{\circ} \mathrm{C}$ to $+8^{\circ} \mathrm{C}$ except with the frozen RAd26-s \& rAd5-s ${ }^{\circledR}$ which requires $-18^{\circ} \mathrm{C}$. AZD1222® may be economical than compared to the other two with at least Rs. $200 /-(2.69 \$)$ per dose as with regard to the expenses behind the logistics for transport through the deeper parts of the country.

Recently new variant B1.617 detected in India (Maharashtra), which is supposed to be more contagious. ${ }^{11}$ It might be the reason for hiking Covid-19 cases rapidly, because there is a possibility that the vaccine uses to induce neutralizing antibodies may not work against this mutant (like South African variant). It is necessary to assess the coverage of vaccine for this variant to know the usefulness of vaccination.

All the vaccine made for immunization were targeting Wuhan-Hu-1 reference virus. ${ }^{10}$ After that lots many variants were detected which developed resistance against vaccines available for immunization. This raises a question, Are we really safe from SARS-CoV-2 infection after vaccination? Believing a vaccine blindly may result in deadly consequences, the main challenge for scientist and researcher is to develop a vaccine which will have equal efficacy for future variants. It will take long duration as well as huge investment, which may not be 
feasible to assure the efficacy within short span of time. Now, the focus of researchers is diverted to induce wide range of immunity by using existing vaccines; therefore, trials of various combinations of the vaccine is ongoing. ${ }^{47}$ As was followed by the manufacturer of RAd26-s \& rAd5-s®, where they have used two different viral vectors i.e., rAd26-s and rAd5-s. ${ }^{43,44,48}$

Table no.1 Head-to-head evaluation of Covid-19 vaccine authorized in India.

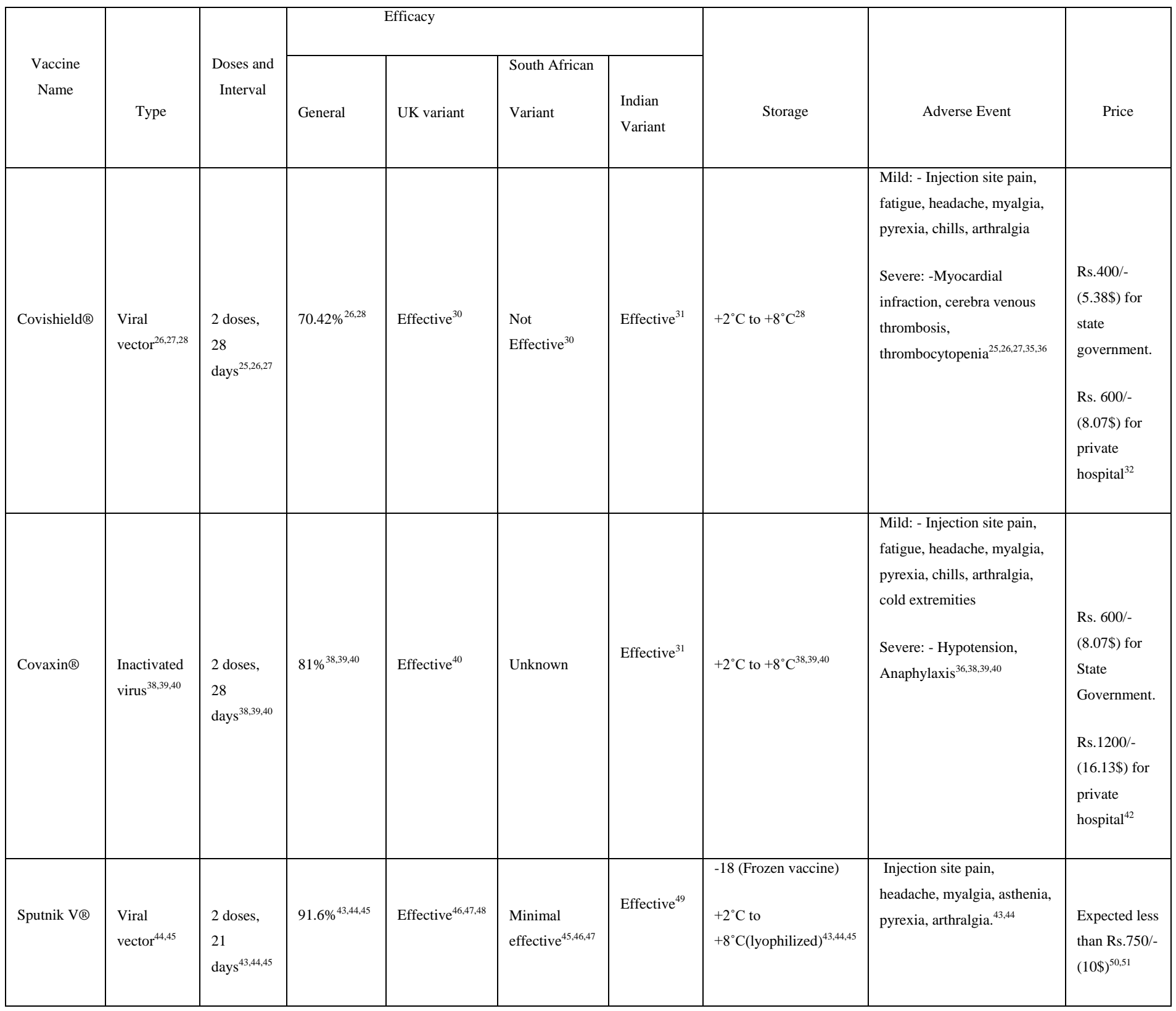

\section{Conclusion:}


It is required to carry out the epidemiological studies to establish a clear endpoint for vaccine efficacy measurement. As per available data, RAd26-s \& rAd5-s V® vaccine is designed as per present interest by using two different viral vectors to induce neutralizing antibodies having wide range of coverage which has shown comparatively greater efficacy in overall (Old and new variants) than BBV152® and AZD1222® vaccines in the prevention of Covid19 and has not associated with serious adverse event, further study may be required, considering new variants.

The main goal of vaccination is to reduce deaths, protect the health care system and finally disease control, this is only be possible if more population is vaccinated with locally available Covid-19 vaccine and self-awareness about the prevention of disease transmission. Wearing mask, maintaining distance, regular hand washing and avoiding crowed is still the best option to prevent the SARS-CoV-2 infection even after vaccination.

Acknowledgements: We would like to thank Dr. B Ramesh, Dean \& principle of Sri Adichunchanagiri College of Pharmacy for his assistance and guidance.

\section{References:}

1. Dai, L., \& Gao, G. F. (2021). Viral targets for vaccines against COVID-19. Nature Reviews Immunology, 21(2), 73-82.

2. Johns Hopkins University (2021). COVID-19 Dashboard by the Center for Systems Science and Engineering (CSSE) at Johns Hopkins University (JHU). Available from: https://coronavirus.jhu.edu/map.html.

3. Lipworth, B., Kuo, C., \& Chan, R. (2020). Emerging pharmacotherapy for COVID19. Journal of the Royal College of Physicians of Edinburgh, 50(2), 133-137.

4. Cheng, H., Wang, Y., \& Wang, G. Q. (2020). Organ-protective effect of angiotensinconverting enzyme 2 and its effect on the prognosis of COVID-19. Journal of medical virology, 92(7), 726-730.

5. Zheng, H., \& Cao, J. J. (2020). ACE gene polymorphism and severe lung injury in patients with COVID-19. The American journal of pathology.

6. Ahlawat, S., \& Sharma, K. K. (2020). Immunological co-ordination between gut and lungs in SARS-CoV-2 infection. Virus research, 198103.

7. Albini, A., Di Guardo, G., Noonan, D. M., \& Lombardo, M. (2020). The SARS-CoV2 receptor, ACE-2, is expressed on many different cell types: implications for ACEinhibitor-and angiotensin II receptor blocker-based cardiovascular therapies. Internal and emergency medicine, 15, 759-766. 
8. Doroftei, B., Ciobica, A., Ilie, O. D., Maftei, R., \& Ilea, C. (2021). Mini-review discussing the reliability and efficiency of COVID-19 vaccines. Diagnostics, 11(4), 579.

9. Srivastava, S., Banu, S., Singh, P., Sowpati, D. T., \& Mishra, R. K. (2021). SARSCoV-2 genomics: An Indian perspective on sequencing viral variants. Journal of Biosciences, 46(1), 1-14.

10. Urhan, A., \& Abeel, T. (2021). Emergence of novel SARS-CoV-2 variants in the Netherlands. Scientific reports, 11(1), 1-15.

11. Everything we know about the Indian COVID-19 variant so far, 21 April 2021. https://www.weforum.org/agenda/2021/04/indian-coronavirus-variant-vaccinescontagious-questions/

12. World Health Organization. The COVID-19 candidate vaccine landscape [Internet]. Geneva, Switzerland: World Health Organization; 2021 [cited 2021 Feb 11]. Available from: https:// www.who.int/publications $/ \mathrm{m} /$ item/draft-landscape-ofcovid?19-candidate-vaccines.

13. Hodgson SH, Mansatta K, Mallett G, Harris V, Emary KR, Pollard AJ. What defines an efficacious COVID-19 vaccine? A review of the challenges assessing the clinical efficacy of vaccines against SARS-CoV-2. The lancet infectious diseases. 2020 Oct 27.

14. US Food and Drug Administration. COVID-19 vaccines [Internet]. Silver Spring, MD: Food and Drug Administration; 2021 [cited 2021 Feb 11]. Available from: https://www.fda.gov/emergency-preparedness-and-response/coronavirus-disease2019-covid-19/covid-19-vaccines.

15. Press Statement by the Drugs Controller General of India (DCGI) on Restricted Emergency approval of COVID-19 virus vaccine. https://www.icmr.gov.in/pdf/press_realease_files/HFW_DCGI_energency_use_autho risation_03012021_2.pdf

16. RAd26-s \& rAd5-s ${ }^{\circledR}$ Covid-19 vaccine gets approval in India: Price, efficacy, how it works: https://www.indiatoday.in/coronavirus-outbreak/story/rAd26-s \& rAd5-s-vcovid-19-vaccine-approval-india-price-storage-availability-1790329-2021-04-13

17. Teo SP. Review of COVID-19 Vaccines and Their Evidence in Older Adults. Annals of Geriatric Medicine and Research. 2021 Mar;25(1):4.

18. Haque A, Pant AB. Efforts at COVID-19 Vaccine Development: Challenges and Successes. Vaccines. 2020 Dec;8(4):739.

19. World Health Organization. COVID-19 vaccines: safety surveillance manual: description and general safety considerations for implementation [Internet]. Geneva, Switzerland: World Health Organization; 2020 [cited 2021 Feb 11]. Available from: https://www.who.int/vaccine_safety/committee/Module_Vaccine_description.pdf?ua $=1$

20. Liu MA. A comparison of plasmid DNA and mRNA as vaccine technologies. Vaccines. 2019 Jun;7(2):37.

21. WHO. WHO target product profiles for COVID-19 vaccines. Geneva: World Health Organization, 2020. 
22. Hodgson SH, Mansatta K, Mallett G, Harris V, Emary KR, Pollard AJ. What defines an efficacious COVID-19 vaccine? A review of the challenges assessing the clinical efficacy of vaccines against SARS-CoV-2. The lancet infectious diseases. 2020 Oct 27.

23. Bose S, Adapa S, Aeddula NR, et al. Medical management of COVID-19: evidence and experience. J Clin Med Res 2020; 12: 329-43.

24. Ministry of information and broadcasting, Government of India. Covid-19 vaccination status. https://mib.gov.in/

25. Folegatti PM, Ewer KJ, Aley PK, Angus B, Becker S, Belij-Rammerstorfer S, Bellamy D, Bibi S, Bittaye M, Clutterbuck EA, Dold C. Safety and immunogenicity of the ChAdOx1 nCoV-19 vaccine against SARS-CoV-2: a preliminary report of a phase 1/2, single-blind, randomised controlled trial. The Lancet. 2020 Aug 15;396(10249):467-78.

26. Voysey M, Clemens SA, Madhi SA, Weckx LY, Folegatti PM, Aley PK, Angus B, Baillie VL, Barnabas SL, Bhorat QE, Bibi S. Safety and efficacy of the ChAdOx1 nCoV-19 vaccine (AZD1222) against SARS-CoV-2: an interim analysis of four randomised controlled trials in Brazil, South Africa, and the UK. The Lancet. 2021 Jan 9;397(10269):99-111.

27. Ramasamy MN, Minassian AM, Ewer KJ, Flaxman AL, Folegatti PM, Owens DR, Voysey M, Aley PK, Angus B, Babbage G, Belij-Rammerstorfer S. Safety and immunogenicity of ChAdOx1 $\mathrm{nCoV}-19$ vaccine administered in a prime-boost regimen in young and old adults (COV002): a single-blind, randomised, controlled, phase 2/3 trial. The Lancet. 2020 Dec 19;396(10267):1979-93.

28. Serum institute of India. AZD1222@-ChAdOx1 nCoV-19 Corona Virus Vaccine Insert, Domestic-New 02012021.cdr https://www.seruminstitute.com/pdf/AZD1222@_ChAdOx1_nCoV19_corona_virus_ vaccine_insert.pdf

29. Karim SS. Vaccines and SARS-CoV-2 variants: the urgent need for a correlate of protection. The Lancet. 2021 Apr 3;397(10281):1263-4.

30. Darton C, Dold C, Douglas AD, Duncan CJ, Ewer KJ, Flaxman A, Faust SN, Ferreira DM, Feng S, Finn A, Folegatti PM. Efficacy of ChAdOx1 nCoV-19 (AZD1222) vaccine against SARS-CoV-2 VOC 202012/01 (B. 1.1. 7).

31. https://www.thehindu.com/news/national/AZD1222-BBV152-made-fewer-antibodiesagainst-b1617-variant/article34560578.ece

32. Serum institute of India pvt.ltd. Media statement April 21. https://www.seruminstitute.com/news_sii_media_210421.php.

33. Scully M, Singh D, Lown R, Poles A, Solomon T, Levi M, Goldblatt D, Kotoucek P, Thomas W, Lester W. Pathologic antibodies to platelet factor 4 after ChAdOx1 nCoV-19 Vaccination. New England Journal of Medicine. 2021 Apr 16.

34. Greinacher A, Thiele T, Warkentin TE, Weisser K, Kyrle PA, Eichinger S. Thrombotic thrombocytopenia after ChAdOx1 nCov-19 vaccination. New England Journal of Medicine. 2021 Apr 9. 
35. Chatterjee S, Ojha UK, Vardhan B, Tiwari A. Myocardial infarction after COVID-19 vaccination-casual or causal?. Diabetes \& Metabolic Syndrome. 2021 Apr 14.

36. Ministry of health and family welfare. Department of health and family welfare. Immunization. Adverse event following immunization reports. https://main.mohfw.gov.in/sites/default/files/immunizationenglish30032021_0.pdf.

37. Ledford H. Scientists probe how a covid vaccine could cause blood clots.Nature. 2021 April 15:592.

38. Ella R, Vadrevu KM, Jogdand H, Prasad S, Reddy S, Sarangi V, Ganneru B, Sapkal G, Yadav P, Abraham P, Panda S. Safety and immunogenicity of an inactivated SARS-CoV-2 vaccine, BBV152: a double-blind, randomised, phase 1 trial. The Lancet Infectious Diseases. 2021 Jan 21.

39. Ella R, Reddy S, Jogdand H, Sarangi V, Ganneru B, Prasad S, Das D, Raju D, Praturi U, Sapkal G, Yadav P. Safety and immunogenicity of an inactivated SARS-CoV-2 vaccine, BBV152: interim results from a double-blind, randomised, multicentre, phase 2 trial, and 3-month follow-up of a double-blind, randomised phase 1 trial. The Lancet Infectious Diseases. 2021 Mar 8.

40. Bharat biotech. BBV152®® - India's First Indigenous COVID-19 Vaccine. Covid fact sheet. https://www.bharatbiotech.com/images/BBV152@/BBV152®-fact$\underline{\text { sheet.pdf }}$

41. Sapkal GN, Yadav PD, Ella R, Deshpande GR, Sahay RR, Gupta N, Mohan VK, Abraham $P$, Panda $S$, Bhargava $B$. Inactivated COVID-19 vaccine BBV152/BBV152® effectively neutralizes recently emerged B 1.1. 7 variant of SARS-CoV-2. Journal of Travel Medicine. 2021 Mar 27.

42. Bharat Biotech fixes BBV152® price at Rs 600/dose for state govts; Rs 1200 for private hospitals, April 24. https://www.cnbctv18.com/healthcare/bharat-biotechBBV152@-price-covid19-vaccination-registration-serum-AZD1222®-price9017941.htm

43. Logunov DY, Dolzhikova IV, Zubkova OV, Tukhvatullin AI, Shcheblyakov DV, Dzharullaeva AS, Grousova DM, Erokhova AS, Kovyrshina AV, Botikov AG, Izhaeva FM. Safety and immunogenicity of an rAd26 and rAd5 vector-based heterologous prime-boost COVID-19 vaccine in two formulations: two open, nonrandomised phase 1/2 studies from Russia. The Lancet. 2020 Sep 26;396(10255):88797.

44. Logunov DY, Dolzhikova IV, Shcheblyakov DV, Tukhvatulin AI, Zubkova OV, Dzharullaeva AS, Kovyrshina AV, Lubenets NL, Grousova DM, Erokhova AS, Botikov AG. Safety and efficacy of an rAd26 and rAd5 vector-based heterologous prime-boost COVID-19 vaccine: an interim analysis of a randomised controlled phase 3 trial in Russia. The Lancet. 2021 Feb 20;397(10275):671-81.

45. Jones I, Roy P. RAd26-s \& rAd5-s® COVID-19 vaccine candidate appears safe and effective. The Lancet. 2021 Feb 20;397(10275):642-3.

46. Healthcare \& Pharmaceuticals. Russia says RAd26-s \& rAd5-s shot less effective vs S.Africa variant but better than others-Ifax. https://www.nasdaq.com/articles/russiasays-rAd26-s \& rAd5-s-shot-less-effective-vs-s.africa-variant-but-better-than-othersifax. 
47. South Africa SARS-CoV-2 variant B.1.351 easily escapes RAd26-s \& rAd5s®accine. https://www.news-medical.net/news/20210406/South-Africa-SARS-CoV2-variant-B1351-easily-escapes-RAd26-s \& rAd5-s-vaccine.aspx.

48. Lawton G. RAd26-s \& rAd5-s® vaccine goes global

49. Russia's RAd26-s \& rAd5-s V vaccine effective against Indian coronavirus strain, says expert. https://tass.com/world/1283807

50. India to get RAd26-s \& rAd5-s® doses in May; 1st batch to be imported from Russia, says Dr Reddy's CEO, 28 April 2021. https://www.businesstoday.in/current/economypolitics/india-to-get-rAd26-s \& rAd5-s-v-doses-in-may-1st-batch-to-be-importedfrom-russia-says-dr-reddy-ceo/story/437726.html

51. The cost of one dose of the RAd26-s \& rAd5-s® vaccine will be less than $\$ 10$ for international markets.

https://rAd26-s

$\&$ rAd5svaccine.com/newsroom/pressreleases/the-cost-of-one-dose-will-be-less-than-10-forinternational-markets/ 